\title{
Quantitative Determination of Hemoglobin in Tooth and Bone by UV/Vis Spectroscopy and Cyclic Voltammetry
}

\author{
Stephanie E Hooper Marosek ${ }^{1 *}$, L Kayci Miller Gowins ${ }^{1}$ and Alice H Suroviec ${ }^{2}$
}

${ }^{1}$ Department of Chemistry and Physical Science, Methodist University, USA

${ }^{2}$ Department of Chemistry, Berry College, USA

\begin{abstract}
Detection and quantification of hemoglobin in skeletal or dried remains could potentially have versatile utilization with the forensic field. However, the majority of studies within this area focus on the identification of hemoglobin and its derivatives rather than determining the relative amount of protein present. This research focuses on the use of leuco crystal violet with Ultraviolet/Visible spectroscopy and applying cyclic voltammetry for the determination and quantification of hemoglobin in a tooth and femur sample. Using leuco crystal violet, possible interferences were also investigated, and it was determined that ferrous iron samples appeared to actually mask the overall reaction, while glycine had no obvious effect. Albumin showed no apparent interference, while myoglobin produced a very faint green color that absorbed at a different wavelength, so this species produced minimal interference. Extractions of hemoglobin from a femur bone using $0.5 \mathrm{M}$ hydrochloric acid and from a tooth using $6.0 \mathrm{M}$ urea all yielded the violet product when reacted with leuco crystal violet. Using the measured absorbance of each positive extraction, the corresponding amount of hemoglobin in each sample was determined. Hemoglobin standards and extraction samples from the tooth and femur bone were also analyzed by the electrochemical method, cyclic voltammetry Hemoglobin levels in the tooth and femur were found to be in the micromolar range in both the spectroscopic and electrochemical analysis, and these levels are consistent with a decrease in hemoglobin concentration postextraction and post-mortem.
\end{abstract}

Keywords: Hemoglobin; Spectroscopic detection; Leuco crystal violet; Electrochemical detection; Bone

\section{Introduction}

Hemoglobin $(\mathrm{Hb})$ is a major oxygen-carrying molecule composed of four globular protein units and four heme moieties that consist of an iron (II) atom held in the surrounding porphyrin ring, which serves as a binding site for oxygen. The four heme groups duplicate the activity of the enzyme horseradish peroxidase [1], and this reactivity plays a critical role for the determination of $\mathrm{Hb}$ in this work. The detection and quantification of $\mathrm{Hb}$ from bone samples could have some potentially useful forensic applications such as helping to establish a time since death, identification of certain health and genetic traits, supporting classification in pink teeth cases, and possible determination of wound age in dry bone fractures. Certain blood proteins such as albumin and $\mathrm{Hb}$ survive well over time within bone [2]. The feasibility of finding $\mathrm{Hb}$ in bone samples of varying age is supported by the ability to detect $\mathrm{Hb}$ breakdown products in a fossilized Tyrannosaurus rex skeleton $[3,4]$. $\mathrm{Hb}$ is well-preserved as it crystallizes easily which may contribute to the stability of the protein, and the rigid structure of heme could possibly add to the durability of the protein [3]. These findings further support the idea that $\mathrm{Hb}$ can be extracted from bone, detected, and quantified.

The majority of studies within this area tend to focus on identifying $\mathrm{Hb}$ and its derivatives from bone samples. Methods such as mass spectrometry, immunoassays, and chemiluminescence have been used to characterize $\mathrm{Hb}$ and other proteins from skeletal remains with varying results. More specifically, two recent studies have focused on the identification of $\mathrm{Hb}$, its derivatives, and related species in bone and teeth samples. Both Pickworth's benzidine method and autofluorescence were utilized to detect $\mathrm{Hb}$ and its derivatives in a case study of shipwrecked remains exhibiting the phenomenon known as pink teeth [5]. Another preliminary study employed certain immunohistological methods to detect hemorrhaging for possible wound age determination in dry bone [6]. Thus far, there has been no quantitative approach to determining $\mathrm{Hb}$ levels in bone or teeth samples. This research is directed at determining the feasibility of such analysis by using a combinatorial approach. The two investigative methods of Ultraviolet/Visible spectroscopy and cyclic voltammetry are employed to detect and quantify $\mathrm{Hb}$ concentrations in a tooth and femur sample. A comparative evaluation of these results will examine any correlation and the advantages or lack thereof for both methods within this study.

$\mathrm{UV} / \mathrm{Vis}$ detection is a simple, common method that has been used for the determination of $\mathrm{Hb}$. Hb itself yields an absorption maximum in the wavelength range of $415-476 \mathrm{~nm}$, but its molar absorptivity is fairly low with a value around $3300 \mathrm{M}^{-1} \mathrm{~cm}^{-1}[7,8]$. A more sensitive approach for detecting $\mathrm{Hb}$ is to react it as a catalyst with a chromogenic reagent such as leuco crystal violet (LCV). LCV is the colorless, entirely reduced form of the triaryl methane dye, crystal violet $\left(\mathrm{CV}^{+}\right)$[9]. LCV is oxidized by hydrogen peroxide in the presence of a biocatalyst such as the heme moiety in $\mathrm{Hb}$. The apparent molar absorptivity of crystal violet formed with LCV in the presence of hydrogen peroxide is $75,600 \pm 298 \mathrm{M}^{-1} \mathrm{~cm}^{-1}$, approximately 20 times greater than the molar absorptivity of $\mathrm{Hb}$ itself [9-11]. LCV is investigated for this research

*Corresponding author: Stephanie E Hooper Marosek, Department of Chemistry and Physical Science, Methodist University, 5400 Ramsey Street, Fayetteville, NC 28311, USA, Tel: 011-1-910-630-7457; Fax: 011-1-910-630-7530; E-mail: shooper@methodist.edu

Received May 11, 2013; Accepted June 14, 2013; Published June 21, 2013

Citation: Hooper Marosek SE, Miller Gowins LK, Suroviec AH (2013) Quantitative Determination of Hemoglobin in Tooth and Bone by UV/Vis Spectroscopy and Cyclic Voltammetry. J Forensic Res 4: 183. doi:10.4172/2157-7145.1000183

Copyright: (c) 2013 Hooper Marosek SE, et al. This is an open-access article distributed under the terms of the Creative Commons Attribution License, which permits unrestricted use, distribution, and reproduction in any medium, provided the original author and source are credited. 
over the use of other reagents such as Amido Black and Luminol for several reasons. LCV itself has a high affinity for proteins, a large molar absorptivity, is fairly safe and easy to work with, and yields extremely sensitive results in a much faster timeframe [12]. The reaction of heme with LCV should provide a more specific, longer response for $\mathrm{Hb}$ in bone than other colorimetric and luminescent methods. Hence, this is the preferred spectroscopic method for determining and quantifying $\mathrm{Hb}$ in bone for this research.

$\mathrm{Hb}$ does exhibit some electrochemical activity, as it contains ferrous iron at the center of each of its four porphyrin rings. The electro-activity of $\mathrm{Hb}$ can be difficult to measure due to its bulky, globular structure and the lengthy pathway electrons must travel from its center to the surface of the working electrode [11]. Most often the slow kinetic redox activity is analyzed through the use of modified electrodes and other enzymatic means. However, if the sample is scanned at a slow enough rate, this enables sufficient electron transfer between the protein and the electrode for a quantitative measurement to be taken. Cyclic voltammetry (CV) is a highly sensitive method that is likely to register any significant electron transfer in to the presence of $\mathrm{Hb}$ due to its dual sweep over a specified potential range. This analysis in combination and comparison with UV/Vis detection should result in selective, sensitive identification and quantification of $\mathrm{Hb}$ in bone and teeth samples.

\section{Materials and Methods}

\section{Solution preparation}

A stock Hb solution (Point Scientific, USA) of $7.447 \times 10^{-3} \mathrm{M}$ was prepared. From this, $\mathrm{Hb}$ standards of $0.25,0.50,1.0,2.0,4.0,10.0$, and $20.0 \mu \mathrm{M}$ were prepared. The stock solution was stored at $4^{\circ} \mathrm{C}$. The LCV solution $(500 \mathrm{~mL})$ was prepared by mixing $150.0 \mathrm{~mL}$ of DI water, $50.0 \mathrm{~mL}$ of $30 \%$ hydrogen peroxide (Fisher Scientific, USA), $10.0 \mathrm{~g}$ of 5-sulphosalicylic acid (Sigma-Aldrich, USA), $4.4 \mathrm{~g}$ of sodium acetate (Fisher Scientific, USA), and $1.1 \mathrm{~g}$ of LCV (99\% pure, Acros, USA). The solution was then brought to volume, inverted 20 times to mix, transferred to an amber bottle, and stored at $4^{\circ} \mathrm{C}$. The following extraction solutions were prepared as well: $0.2 \mathrm{M}$ Tris buffer (SigmaAldrich, USA), $0.5 \mathrm{M} \mathrm{HCl}$ (Fisher Scientific, USA), and a $6.0 \mathrm{M}$ urea solution (Amresco, USA).

\section{Bone and tooth preparation and extraction}

A tooth sample (1-2 years in age post-extraction) was sawed into four different sections, and then sanded down to obtain powder from the innermost portion of the tooth. A femur bone (5-10 years in age post-mortem) was sawed in half at the head and the shaft of the bone and powder was collected from the inner cortical bone (Figure 1). Eleven samples were prepared containing $\sim 0.25$ grams of powder from the tooth, femur head, or femur shaft and $1.5 \mathrm{~mL}$ of one of the 3 extraction solvents. The samples were shaken well every $10 \mathrm{~min}$. for 1 hour. Each sample then had 2 drops of LCV added, and was stored at $4^{\circ} \mathrm{C}$ overnight. The resulting samples were:

Tooth in (A) $50 \% 0.5 \mathrm{M} \mathrm{HCl} / 50 \%$ DI Water, (B) $0.5 \mathrm{M} \mathrm{HCl}$, (C) $50 \% 0.2 \mathrm{M}$ Tris Buffer/50\% DI Water, (D) $0.2 \mathrm{M}$ Tris Buffer, and (K) 6.0 M Urea.

Femur shaft in (E) 6.0 M Urea, (F) 0.5 M HCl, (G) 0.2 M Tris Buffer

Femur head in (H) 6.0 M Urea, (I) 0.5 M HCl, (J) 0.2 M Tris Buffer

\section{Preliminary tests with leuco crystal violet}

As a preliminary test, solutions of varying $\mathrm{Hb}$ concentrations were used to determine if LCV would successfully detect the presence of $\mathrm{Hb}$. Upon the addition of two drops of LCV, each standard turned a varying shade of purple, a positive indication for the presence of $\mathrm{Hb}$. The standards with LCV were allowed to sit overnight at $4^{\circ} \mathrm{C}$. The violet color remained in these solutions for up to 48 hours, demonstrating the stability of the $\mathrm{Hb} / \mathrm{LCV}$ reaction product. However, fresh standards were prepared daily to maintain a proper working calibration curve. $\mathrm{Hb}$ was initially detected, so corresponding absorption data was then collected.

\section{UV/Vis spectroscopy}

Spectroscopic analysis was performed on a midrange $\mathrm{Hb}$ standard $(1.0 \mu \mathrm{M})$ containing two drops of LCV. The absorption spectrum for this $\mathrm{Hb}$ standard as shown in Figure 2 yielded a maximum wavelength value of $590 \mathrm{~nm}$. The spectrum was obtained using an Ocean Optics (USA) Red Tide USB650 Spectrometer with a $1 \mathrm{~cm}$ path length. Subsequent analysis for all standards and extraction results was performed on a Spectronic (UK) 20 Genesys set to a $\lambda_{\max }$ of $590 \mathrm{~nm}$ using a $1 \mathrm{~cm}$ path length.

Spectroscopic measurements were taken three times for each standard (average $s$ of \pm 0.002 Abs. units) and sample (average $s$ of \pm $0.002 \mathrm{Abs}$. units), and the corresponding average values were utilized for data analysis and interpretation.

\section{Interferences}

The effect of interferences such as ferrous iron, glycine, albumin, and myoglobin possibly masking or enhancing the response for $\mathrm{Hb}$ in the presence of LCV was investigated. The presence of ferrous iron $\left(\mathrm{Fe}^{+2}\right)$ in skeletal remains is often an indication of dietary behavior,

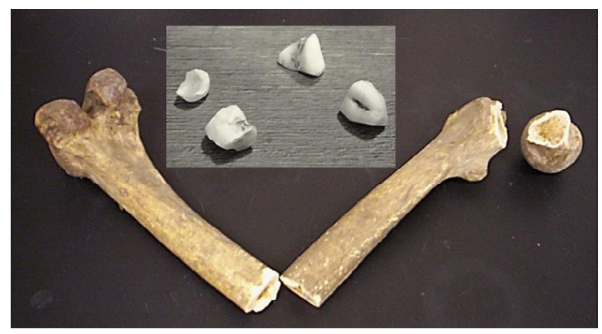

Figure 1: The tooth sample (inset), femur shaft, and femur head used for hemoglobin extraction and spectroscopic analysis.

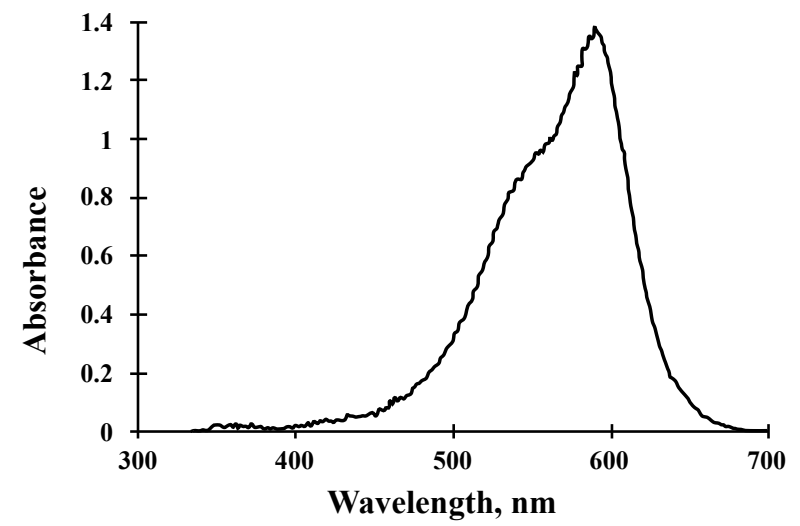

Figure 2: The UV/Vis absorbance spectrum of the LCV reaction with 1.0 $\mu \mathrm{M} \mathrm{Hb}$. 
which could lead to a false response for $\mathrm{Hb}$ with LCV [13]. Glycine is an amino acid used for a variety of health reasons including the treatment of iron deficiency anemia [14,15]. Glycine chelates iron, so its effect on the $\mathrm{Hb}$ response to LCV was also studied. A few granules of ferrous ammonium sulfate, $\left(\mathrm{NH}_{4}\right)_{2} \mathrm{Fe}\left(\mathrm{SO}_{4}\right)_{2} \cdot 6 \mathrm{H}_{2} \mathrm{O}$, Fisher Scientific, USA), ferrous sulfate, $\left(\mathrm{FeSO}_{4}\right.$ Mallinckrodt Baker, USA) and glycine (Fisher Scientific, USA) were tested on the $1.0 \mu \mathrm{M} \mathrm{Hb}$ standard with LCV. The results of these interferences on the reaction of $\mathrm{Hb}$ and LCV can be seen in Figure 3. Ferrous ammonium sulfate immediately reacted to reduce the purple color to approximately one-third of the original color. Ferrous sulfate reacted to leave approximately two-thirds of the purple color. Glycine had no obvious effect on the solution. From these observations, these species do not result in a darker product with LCV, so false positives appear to be minimal, if not unlikely.

Albumin is a blood protein most abundant in human plasma, and it has a similar structure and molecular weight to hemoglobin. Myoglobin is also a blood protein that contains a heme moiety, so the LCV could possibly react and produce an interfering purple color if this species is present in a particular sample being analyzed. Solutions of $1.0 \mu \mathrm{M}$ albumin (Protea Biosciences, USA) and myoglobin (Protea Biosciences, USA) were prepared in deionized water and treated with two drops of LCV. The results of these reactions can also be seen in Figure 3. Albumin remained a clear solution indicating it will not interfere with the reaction of $\mathrm{Hb}$ and LCV. Myoglobin reacted to produce a very faint green colored solution, which indicates there may be a slight interference in the presence of this particular protein, but not of a significant nature. This faint green product also absorbed at a wavelength different from the $\lambda_{\max }$ of $590 \mathrm{~nm}$ for LCV. A simple background correction eliminated the interference response of myoglobin with LCV.

\section{Electrochemistry}

Cyclic voltammetry measurements were performed using a $\mathrm{CH}$ Instruments (USA) 800B Electrochemical Analyzer. The threeelectrode system utilized a $2 \mathrm{~mm}$ gold disk working electrode $(\mathrm{CH}$ Instruments, USA), a $\mathrm{Ag} / \mathrm{AgCl}$ reference electrode ( $\mathrm{CH}$ Instruments, USA), and a $0.5 \mathrm{~mm}$ Platinum auxiliary wire (Alfa Aesar, USA). Hb standards and samples were prepared in $0.05 \mathrm{M}$ phosphate buffer and the appropriate extraction solution, and subsequently analyzed at a scan rate of $0.005 \mathrm{~V} / \mathrm{s}$. Scan rates faster than this value did not yield as high peak currents for each standard and sample, so the aforementioned rate was determined to be optimal.

$\mathrm{CV}$ measurements were run a total of three times for all standards (average $s$ of $\pm 9.3 \times 10^{-8} \mathrm{~A}$ ) and samples (average $s$ of $\pm 8.9 \times 10^{-8} \mathrm{~A}$ ), and the corresponding average values were utilized for data analysis.

\section{Results and Discussion}

The ultimate objective of this research was to detect and quantify $\mathrm{Hb}$ in tooth and bone samples by spectroscopic measurement using LCV and electrochemical analysis using cyclic voltammetry. Three different extraction solutions $(0.5 \mathrm{M} \mathrm{HCl}, 0.2 \mathrm{M}$ Tris, and $6.0 \mathrm{M}$ Urea) were used to extract and detect $\mathrm{Hb}$ in tooth, femur head, and femur shaft samples. The use of these solutions was based on previous extraction studies $[16,17]$. Positive results for the following three samples were obtained: the femur shaft sample in $0.5 \mathrm{M} \mathrm{HCl}(\mathrm{F})$, the femur head sample in 0.5 $\mathrm{M} \mathrm{HCl}(\mathrm{I})$, and the tooth sample in $6.0 \mathrm{M}$ urea (K). Each of these three samples contained extracted $\mathrm{Hb}$ that was detectable with the LCV by UV/Vis spectroscopy (Figure 4) and by cyclic voltammetry (Figure 5).

To quantify these positive results spectrophotometrically with

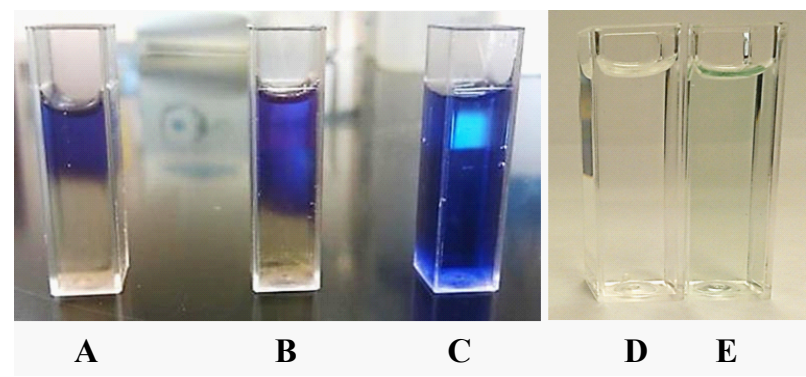

Figure 3: The effects of interferences on the reaction of $\mathrm{Hb}$ and $\mathrm{LCV}$ : ferrous ammonium sulfate, (B) ferrous sulfate, (C) glycine, (D) albumin, and (E) myoglobin

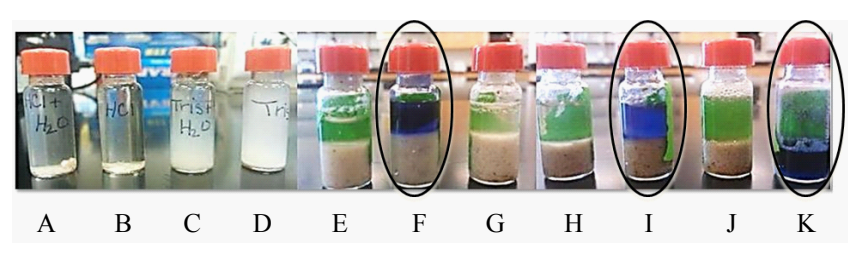

Figure 4: Extraction samples from left to right: tooth in (A) $50 \% 0.5 \mathrm{M} \mathrm{HCl}$ $150 \%$ DI Water, (B) $0.5 \mathrm{M} \mathrm{HCl}$, (C) $50 \% 0.2 \mathrm{M}$ Tris Buffer/50\% DI Water, (D) $0.2 \mathrm{M}$ Tris Buffer, femur shaft in (E) $6.0 \mathrm{M}$ Urea, $(\mathrm{F}) 0.5 \mathrm{M} \mathrm{HCl},(\mathrm{G}) 0.2 \mathrm{M}$ Tris Buffer, femur head in $(\mathrm{H}) 6.0 \mathrm{M}$ Urea, (I) $0.5 \mathrm{M} \mathrm{HCl},(\mathrm{J}) 0.2 \mathrm{M}$ Tris Buffer, and tooth in (K) 6.0 M Urea.

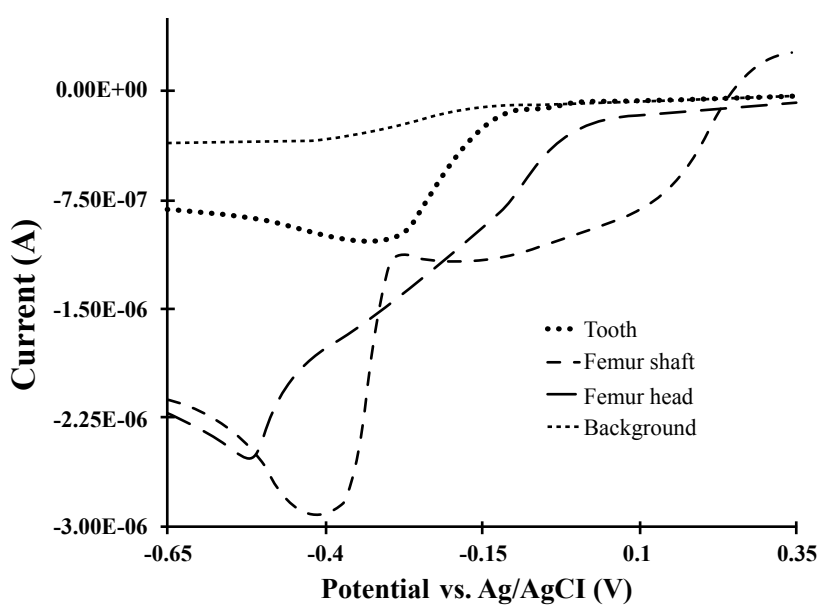

Figure 5: The cyclic voltammogram of hemoglobin extracted from the tooth and femur samples along with the background scan. The faradaic current was measured at the corresponding reduction potential peak for each sample.

$\mathrm{LCV}, \mathrm{Hb}$ standards were prepared and a calibration curve was obtained. An $\mathrm{Hb}$ concentration of $14.9 \mathrm{nM}$ was determined to establish the limit of quantification for this set of measurements. Absorbance measurements for the three positive samples from the tooth and femur were obtained. By using the slope equation from the calibration curve, the $\mathrm{Hb}$ concentrations for each of the three positive samples were calculated. The $\mathrm{Hb}$ concentrations for each sample are given in Table 1. These three samples are all less concentrated than the normal $\mathrm{Hb}$ levels of a human, approximately $2 \mathrm{mM}$, which supports that $\mathrm{Hb}$ levels in bone decrease over time post-mortem and post-extraction. 


\begin{tabular}{|l|l|l|}
\hline Bone Sample & $\begin{array}{l}\text { Hb Concentration }(\boldsymbol{\mu M}) \\
\text { from UV/Vis Analysis }\end{array}$ & $\begin{array}{l}\text { Hb Concentration }(\boldsymbol{\mu M}) \\
\text { from CV Analysis }\end{array}$ \\
\hline Tooth $(6 \mathrm{M}$ Urea) & 2.60 & 0.47 \\
\hline Femur Shaft $(0.5 \mathrm{M} \mathrm{HCl})$ & 2.13 & 0.59 \\
\hline Femur Head $(0.5 \mathrm{M} \mathrm{HCl})$ & 0.85 & 0.94 \\
\hline
\end{tabular}

Table 1: Tooth and bone sample concentration results for UV/Vis and Voltammetry Analysis.

The results obtained electrochemically were less sensitive than the spectroscopic method using LCV. A higher limit of quantification of $241 \mathrm{nM}$ was established for this particular analysis, which is most likely due to the slow kinetic electron transfer. The bulky nature of the $\mathrm{Hb}$ molecule limits the mobility of electrons towards the working electrode surface. Utilizing the data from the voltammetric calibration curve, levels of $\mathrm{Hb}$ were determined for the three positive extraction sample as shown in Table 1. These sample concentrations also show a similar decrease in $\mathrm{Hb}$ both post-mortem and post-extraction.

These $\mathrm{Hb}$ concentration values illustrate that although the spectroscopic method using LCV yields lower limits of quantification for $\mathrm{Hb}$, there is no sample modification necessary for electrochemical analysis, so samples could be further utilized for immunoassay or DNA testing. The combination of these two techniques demonstrates that $\mathrm{Hb}$ levels in tooth and bone can be quantified, and there is a significant decrease in $\mathrm{Hb}$ concentration post-mortem and post-extraction. While there is some discrepancy among the $\mathrm{Hb}$ values determined for both methods, each analysis method shows the feasibility of quantifying the protein within skeletal samples. Although these techniques demonstrate that quantification of $\mathrm{Hb}$ in bone and teeth is possible, further research with a variety of samples ranging in age post-mortem would be necessary to establish any specific correlation between $\mathrm{Hb}$ levels and a time since death or other forensic phenomena.

The measurement of $\mathrm{Hb}$ levels in bone makes this work unique, as the majority of studies focus solely on the identification of $\mathrm{Hb}$ and related species in bone and teeth, not on quantification. These methods might be potentially useful in certain cases where the ability to quantify $\mathrm{Hb}$ concentrations in tooth and bone samples would be advantageous. The detection method involving LCV is preferable to other colorimetric and luminescent reagents due to its sensitivity, stability, simplicity, and safety. The purple reaction product can last several hours, as opposed to very short-lived reactions with reagents such as luminol. LCV itself is relatively safe to work with compared to hazardous reagents like ninhydrin (a known toxin) and benzidine (a known carcinogen). Moreover, $\mathrm{Hb}$ within the samples is not altered and could be used for further analysis by certain electrochemical methods. This is because the catalyst, $\mathrm{Hb}$, does not actually take part in the reaction. The voltammetric results here demonstrate that detection of $\mathrm{Hb}$ is possible with these extraction methods, with a slight sacrifice in overall sensitivity.

The use of LCV is slightly destructive to the extraction sample, so this method does limit the amount of biological and immunological testing possible. This drawback can be overcome by the use of cyclic voltammetry as a quantification method for $\mathrm{Hb}$, because no modification of the extraction sample is required. Another small limitation is the possibility of false positives, but these appear to be minimal for this particular analysis. Environmental factors, age, and circumstantial variables could play a role in the subsequent sensitivity for the quantitation method, but the specificity and relative stability of the LCV reaction product and the electron transfer process in cyclic voltammetry should not be compromised. Overall, these results demonstrate that UV/Vis spectroscopy using LCV and electrochemical analysis are capable of providing specific and quantitative detection of $\mathrm{Hb}$ in bone and teeth. These methods would be mutually advantageous if conjunction with other methods such as auto-fluorescence and immunohistology.

\section{Conclusion}

Quantitative determination of hemoglobin $(\mathrm{Hb})$ in skeletal or dried remains could potentially have a wide range of forensic applications. There have been successful studies illustrating that blood proteins such as certain $\mathrm{Hb}$ components can be recovered and identified from fossilized or skeletal remains. This research focused on the use of LCV with UV/Vis spectroscopy and cyclic voltammetry for the detection and quantification of $\mathrm{Hb}$ in tooth and femur samples. The reaction of LCV and hydrogen peroxide with the heme group of Hb yields a deep violet color. Using LCV, possible interferences were investigated, and it was determined that ferrous samples appear to reduce the $\mathrm{CV}^{+}$back to LCV, while glycine did not appear to affect the reaction with $\mathrm{Hb}$. A slight interference by myoglobin was overcome by implementing a background correction, while albumin did not appear to hinder the reaction of $\mathrm{Hb}$ with $\mathrm{LCV}$. $\mathrm{Hb}$ was successfully extracted from tooth and femur bone samples and was subsequently quantified using the reaction of LCV spectroscopically and by electrochemical analysis. The extractions of the femur head and shaft with $0.5 \mathrm{M} \mathrm{HCl}$ and the extraction of the tooth with $6.0 \mathrm{M}$ urea yielded measurable amounts of $\mathrm{Hb}$ given in Table 1, all of which are consistent with a decrease in concentration post-mortem and post-extraction. Thus, Hb levels in bone and tooth samples were detected and quantified by both methods. While these particular results have not yet established any direct relationship between $\mathrm{Hb}$ levels in human remains and a post-mortem interval or other phenomena, the combination of these two analytical techniques illustrates the possibilities for various applications of quantifying $\mathrm{Hb}$ in bone and teeth. Future research would focus on the application of these methods to a wider variety of bone samples ranging in morphology, age, and condition, while minimizing some of the possible limitations within this method.

\section{Ackowledgement}

The tooth and the femur were obtained from Dr. Bryan Brendley of the Department of Biology at Guilford College in Greensboro, NC.

\section{References}

1. Introna F Jr, Di Vella G, Campobasso CP (1999) Determination of postmortem interval from old skeletal remains by image analysis of luminol test results. $J$ Forensic Sci 44: 535-538.

2. Cattaneo C, Gelsthorpe K, Phillips P, Sokol RJ (1992) Reliable identification of human albumin in ancient bone using ELISA and monoclonal antibodies. Am J Phys Anthropol 87: 365-372.

3. Schweitzer MH, Marshall M, Carron K, Bohle DS, Busse SC, et al. (1997) Heme compounds in dinosaur trabecular bone. Proc Natl Acad Sci USA 94: 6291-6296.

4. Schweitzer MH, Suo Z, Avci R, Asara JM, Allen MA, et al. (2007) Analyses of soft tissue from Tyrannosaurus rex suggest the presence of protein. Science 316: $277-280$.

5. Campobasso CP, Di Vella G, De Donno A, Santoro V, Favia G, et al. (2006) Pink teeth in a series of bodies recovered from a single shipwreck. Am J Forensic Med Pathol 27: 313-316.

6. Cattaneo C, Andreola S, Marinelli E, Poppa P, Porta D, et al. (2010) The detection of microscopic markers of hemorrhaging and wound age on dry bone: a pilot study. Am J Forensic Med Pathol 31: 22-26.

7. Hempe JM, Craver RD (2000) Separation of hemoglobin variants with similar charge by capillary isoelectric focusing: value of isoelectric point for identification of common and uncommon hemoglobin variants. Electrophoresis 21: 743-748. 
8. Zijlstra WG, Buursma A, Meeuwsen-van der Roest WP (1991) Absorption spectra of human fetal and adult oxyhemoglobin, de-oxyhemoglobin, carboxyhemoglobin, and methemoglobin. Clin Chem 37: 1633-1638.

9. Mottola HA, Simpson BE, Gorin G (1970) Absorptiometric Determination of Hydrogen Peroxide in Submicrogram Amounts with Lueco Crystal Violet and Peroxidase as Catalyst. Anal Chem 42: 410-411.

10. Gere EP, Bérczi B, Simándi P, Wittmann G, Dombi A (2002) Simultaneous Determination of Hydrogen Peroxide and Organic Hydroperoxides in Water. Intern J Environ Anal Chem 82: 443-450.

11. Bodziak WJ (1996) Use of leuco crystal violet to enhance shoe prints in blood Forensic Sci Int 82: 45-52.

12. Dai Z, Liu S, Ju H, Chen H (2004) Direct electron transfer and enzymatic activity of hemoglobin in a hexagonal mesoporous silica matrix. Biosens Bioelectron 19: $861-867$

13. Boyer R (2002) Concepts in Biochemistry. (2nd edn) Brooks/Cole, Pacific Grove, CA,USA

14. Hopkins S (1979) Controlled iron release tablets. Drugs of Today 15: 404-406.

15. Jin YS, Lai JQ, Zhao XF, Meng J, Yin S (2005) Evaluation on glycine chelated iron (II) in improving nutritional anemia in rats. Wei Sheng Yan Jiu 34: 344-346.

16. Ascenzi A, Brunori M, Citro G, Zito R (1985) Immunological detection of hemoglobin in bones of ancient Roman times and of Iron and Eneolithic Ages. Proc Natl Acad Sci USA 82: 7170-7172.

17. Jiang X, Ye M, Jiang X, Liu G, Feng S, et al. (2007) Method development of efficient protein extraction in bone tissue for proteome analysis. J Proteome Res 6: 2287-2294. 